\title{
Comprehensive approaches for diagnosis, monitoring and treatment of chronic inflammatory demyelinating polyneuropathy
}

Anna Lena Fisse ${ }^{1,2+}$, Jeremias Motte ${ }^{1,2^{*+}} \mathbb{B}$, Thomas Grüter ${ }^{1,2}$, Melissa Sgodzai ${ }^{1,2}$, Kalliopi Pitarokoili, ${ }^{1,2}$ and Ralf Gold ${ }^{1,2}$

\begin{abstract}
Chronic inflammatory demyelinating polyradiculoneuropathy (CIDP) is the most common chronic inflammatory neuropathy. CIDP is diagnosed according to the European Federation of Neurological Societies/Peripheral Nerve Society (EFNS/PNS) criteria, which combine clinical features with the electrophysiological evidence of demyelination. However, firstly, diagnosis is challenging, as some patients e.g. with severe early axonal damage do not fulfil the criteria. Secondly, objective and reliable tools to monitor the disease course are lacking. Thirdly, about 25\% of CIDP patients do not respond to evidence-based first-line therapy. Recognition of these patients is difficult and treatment beyond first-line therapy is based on observational studies and case series only. Individualized immunomodulatory treatment does not exist due to the lack of understanding of essential aspects of the underlying pathophysiology. Novel diagnostic imaging techniques and molecular approaches can help to solve these problems but do not find enough implementation. This review gives a comprehensive overview of novel diagnostic techniques and monitoring approaches for CIDP and how these can lead to individualized treatment and better understanding of pathophysiology.
\end{abstract}

Keywords: Chronic inflammatory demyelinating polyneuropathy, Inflammatory neuropathies, Imaging, Pathophysiology, Diagnosis, Monitoring, Treatment, Register, Biobank

\section{Background}

The chronic inflammatory demyelinating polyradiculoneuropathy (CIDP) is the most common chronic inflammatory neuropathy. Chronic and recurrent polyneuritis was first described in 1890 by Eichhorst (Eichhorst H.: Polyneuritis recurrens. Correspondenzblatt f. Schweizer Ärzte 1890, publication not digitally available). Around 1950 reports about steroid responsive chronic polyneuritis arose. The term 'chronic inflammatory demyelinating

\footnotetext{
* Correspondence: Jeremias.Motte@rub.de

${ }^{\dagger}$ Anna Lena Fisse and Jeremias Motte contributed equally to this work.

'Department of Neurology, St. Josef-Hospital, Ruhr-University Bochum, Gudrunstrasse 56, 44791 Bochum, Germany

${ }^{2}$ Immunmediated Neuropathies Biobank (INHIBIT), Ruhr-University Bochum, Bochum, Germany
}

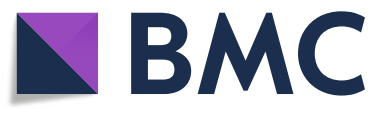

() The Author(s). 2020 Open Access This article is licensed under a Creative Commons Attribution 4.0 International License, which permits use, sharing, adaptation, distribution and reproduction in any medium or format, as long as you give

appropriate credit to the original author(s) and the source, provide a link to the Creative Commons licence, and indicate if changes were made. The images or other third party material in this article are included in the article's Creative Commons licence, unless indicated otherwise in a credit line to the material. If material is not included in the article's Creative Commons licence and your intended use is not permitted by statutory regulation or exceeds the permitted use, you will need to obtain permission directly from the copyright holder. To view a copy of this licence, visit http://creativecommons.org/licenses/by/4.0/. et al. 1982 [1]. CIDP is a relapsing-remitting or progressive inflammatory neuropathy with a multifaceted presentation. There are multiple other chronic inflammatory neuropathies besides CIDP. In the past decades several diagnostic criteria for diagnosis of CIDP were established. The European Federation of Neurological Societies/Peripheral Nerve Society (EFNS/PNS) criteria [2] published in 2006 and revised in 2010, were validated in a multicenter European cohort and have since been broadly adopted in special for clinical trials. They combine clinical features with the electrophysiological evidence of demyelination. Despite these criteria misdiagnosis of CIDP is a problem. 
About 25\% of CIDP patients do not respond to evidence-based first-line therapy with steroids, plasma exchange and intravenous immunoglobulins. Individualized immunomodulatory treatment does not exist due to the lack of understanding of essential aspects of the underlying pathophysiology. Definition of treatment response is often difficult, as objective and reliable tools to monitor the disease course are lacking. This review gives a comprehensive overview of diagnosis, monitoring and treatment as well as pathophysiology of CIDP.

\section{The challenge of correct diagnosis and lucid terminology}

Prevalence of CIDP is estimated between 0.8 to 8.9 cases per 100,000 [3-5]. Typically, more men than women are affected $(2: 1)$, and mean age is about 40 50 years [3-5]. Regional differences of prevalence between continents as known for acute inflammatory demyelinating polyneuropathies are not known for CIDP, as systematic data on epidemiology are lacking [6]. Dietary habits and antecedent infections may have an impact on the risk, onset and clinical presentation of the disease [7].

The challenge of the correct diagnosis is depicted through the fact that more than 15 sets of diagnostic criteria were used over the last 50 years $[8,9]$. The currently most widely accepted criteria, the EFNS/PNS criteria, were established in 2005, revised in 2010 [2]. They combine clinical criteria with electrophysiological evidence of demyelination, while the evidence of inflammation is only included in the supportive criteria through consideration of nerve biopsy and magnetic resonance imaging (MRI). Clinically, EFNS/PNS criteria differentiate typical CIDP with proximal and distal weakness and sensory dysfunction of all extremities from atypical CIDP, in which predominantly distal, asymmetric or focal, pure sensory or pure motor symptoms occur. Supportive criteria also include elevation of protein in cerebrospinal fluid (CSF), response to treatment and abnormal sensory electrophysiology in at least one nerve. Additionally, laboratory exclusion of other conditions is demanded for correct diagnosis.

However, there are some limitations of EFNS/PNS criteria described below. Electrophysiological criteria are complex and extensive and therefore difficult to use in daily clinical practice. The use of incomplete electrophysiological protocols can lead to misdiagnoses and delayed diagnoses [9]. Also, clinical experience reveals patients i.e. with predominant axonal damage who do not fulfil electrophysiological criteria, although they probably have an inflammatory neuropathy.

The supportive criteria include some further difficulties as well. Breiner et al. suggested age-dependent cut-off values [10] for elevation of protein in CSF with a sensitivity of $80-90 \%$ and specificity of $50-60 \%$, but the optimal cut- off value to avoid overdiagnosis is unclear [9]. Also, the role and right timepoint of nerve biopsy in detection of inflammatory infiltrates and demyelination compared to electrophysiological studies remains unknown [11-13]. MRI is difficult to use in everyday practice, due to the required technical expertise in specific imaging protocols and costs. Treatment response as a supportive criterion is not defined and challenging to objectify, possibly leading to overdiagnosis. Moreover, knowledge about pathophysiology of distinct subgroups like nodo- and paranodopathies is not yet represented in EFNS/PNS criteria.

Studies on somatosensory evoked potentials (SEP) to detect demyelination in CIDP showed that SEP are an useful additional tool to NCS [14]. Therefore, SEP are part of the EFNS/PNS additional criteria. However, in daily clinical practice, SEP may be time consuming and technically difficult to analyze and therefore do not play a major role.

Clinical definition of atypical CIDP mentioned in EFNS/PNS criteria is vague. 2018 Doneddu et al. [15] defined more specific and detailed criteria for atypical CIDP. Described subtypes are distal acquired demyelinating symmetric neuropathy (DADS) without proximal limb-trunk-face involvement, pure sensory CIDP without weakness and Lewis-Sumner syndrome with a multifocal distribution of symptoms, also called multifocal acquired demyelinating sensory and motor neuropathy (MADSAM). Again, these definitions are based on the clinical and electrophysiological aspects only and do not consider pathophysiology or novel imaging techniques. Definitions of Doneddu et al. partly seem to be somewhat rigid as patients may change from one clinical subtype to another during their disease course. A more precise characterization of atypical CIDP, both clinically and paraclinically, as well as consensus criteria for atypical CIDP are still lacking. As a result, misdiagnosis of CIDP is common, especially in patients that are classified as atypical CIDP.

There are multiple other chronic inflammatory neuropathies besides CIDP with distinct pathophysiology such as multifocal motor neuropathy (MMN), paraproteinemic demyelinating neuropathies (PDN) with and without anti-MAG (Myelin-associated glycoprotein) antibodies as well as nodo- and paranodopathies. Further entities like MADSAM and DADS are defined as subgroups of CIDP but also have distinct clinical characteristics, treatment response and probably distinct pathophysiology. As the terminology of CIDP subgroups therefore seems to be heterogenous, the term 'chronic inflammatory neuropathies' (CIN) has been used in order to summarize all these entities [6] (Fig. 1). On the other hand, differentiation of subgroups and not lumping all entities together is necessary to enable individualized treatment and better understanding of pathophysiology. 


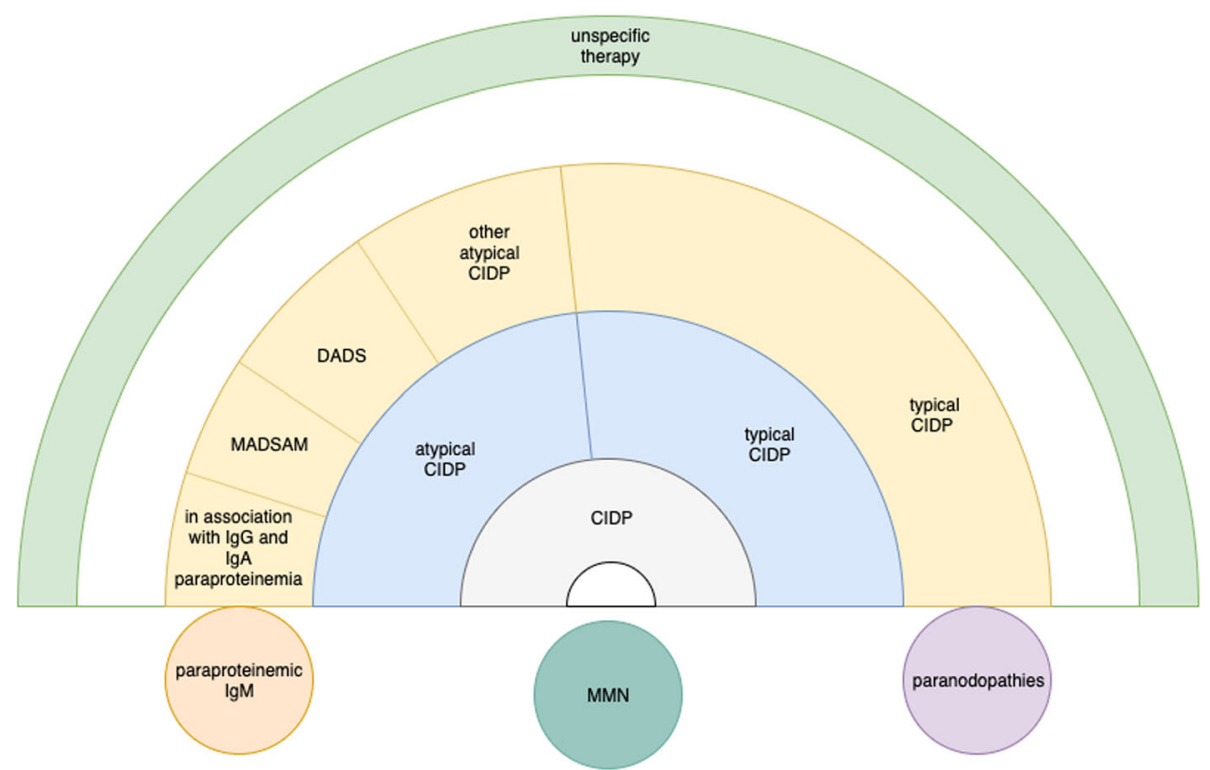

Fig. 1 Overview of inflammatory neuropathies with focus on CIDP, subtypes and distinct disease entities

\section{The challenge of clinical and electrophysiological monitoring}

Classic methods for monitoring CIDP are clinical course and electrophysiology. Disability and symptom scores enable precise clinical characterization and comparison of symptoms in disease course in an objective manner.

- The Medical Research Council (MRC) sum score, originally developed for Guillain-Barré-Syndrome (GBS) patients in the 1970ies, is part of the standard repertoire of clinical examinations used to record muscle strength [16].

- The INCAT-Overall Disability Sum Score (ODSS), first described in 2002, is well-established and validated for patients with CIDP and has developed into a standard score for CIDP [17]. Yet, this score poorly detects discrete changes of disability or sensory symptoms.

- The INCAT sensory sumscore (ISS) is one of the few scores that sensitively records sensory symptoms in patients with GBS and CIDP [18].

- The Rasch-built Overall Disability Scale (R-ODS) is an improved disability score validated for CIDP, GBS and polyneuropathy associated with monoclonal gammopathy of unclear significance (MGUS). Indeed, it enables detection of minor changes compared to the INCAT-ODSS $[19,20]$.

In the recent years, symptoms other than sensorimotor impairment like quality of life [21] were in focus. Further symptoms like pain and fatigue need to be addressed in future studies.

A bedside tool to monitor grip strength is the Martin Vigorimeter which was shown to be a reliable and responsive tool in CIDP patients [22].

It appears obvious that a worsening of the disease can be depicted by nerve conduction studies. However, nerve conduction studies cannot reproduce clinical dynamics, i.e. due to severe secondary axonal damage [23]. Studies on use of electromyography for disease monitoring are lacking. Recently we described that evidence of persistent spontaneous denervation activity could display disease activity (own work under review). Yet, electromyography is invasive, painful and contraindications may prevent regular use. Therefore, use of electrophysiology for monitoring of CIDP is limited although there is extensive knowledge for many generations of clinical neurologists.

\section{Peripheral nerve and muscle imaging as novel diagnostic approaches \\ MRI}

Morphologic alterations of nerves can be detected by MRI. Its main advantages are high-resolution and ability to image deep and proximal tissues. However, differences in acquisition and analysis may result in significant limitations regarding validity. Short tau inversion recovery (STIR) sequences and nerve-specific T2-weighted magnetic resonance neurography (MRN) are used to quantify hypertrophy and depict increased signal intensity as signs of inflammation. Diffusion tensor imaging 
(DTI) enables evaluation of microstructural integrity using the parameter of fractional anisotropy, which indicates demyelination [24, 25]. However, these novel techniques are only used in selected patients or as part of cross-sectional studies. Broadly available MRI rather enables imaging of spinal roots and brachial and lumbosacral plexus to depict hypertrophy and gadolinium-enhancement which is represented as supportive criterion in EFNS/PNS criteria.

\section{Nerve ultrasound}

Nerve ultrasound also enables a non-invasive view of morphology of affected peripheral nerves. The benefit of nerve ultrasound in the diagnosis of CIDP has been proven several times over the past 7 years, but it is still not established as standard diagnostic criterion. The measurement of cross-sectional area (CSA) in ultrasound correlates well to CSA detected by MRI and with the nerve T2-weighted signal intensity [26-28]. Morphological changes like swollen, hypoechogenic nerve and fascicles detected in ultrasound represent acute inflammation [29, 30], while hyperechogenic nerves rather are supposed to occur in case of fibroid remodeling and axonal damage $[29,31]$. Thus, measurement of CSA on the one hand, and assessment of the echogenicity on the other hand are the main parameters for assessing CIDP by ultrasound. Figure 2 exemplarily shows an ultrasound image of a normal median nerve at the forearm of a healthy person and a significantly enlarged nerve of a CIDP patient.
CSA enlargements can occur focally, multifocally or more generalized. Frequently, not only enlarged CSA of the whole nerve, but also individual enlarged fascicles can be observed in some CIDP patients. Multiple publications have shown the value of HRUS as an add-on tool to electrophysiological examinations for the diagnosis of CIDP and several ultrasound protocols, normal values and scores based on CSA were published to diagnose CIDP and differentiate it from GBS as well as differentiation protocols for atypical CIDP forms [32-35]. Published nerve ultrasound protocols distinguish acute and chronic inflammatory polyneuropathies as well as hereditary polyneuropathies $[33,36]$. It has not yet been investigated, whether nerve ultrasound also helps to distinguish axonal non-inflammatory polyneuropathies from CIDP with secondary axonal damage.

The correlation between a morphologically focal swollen nerve observed by ultrasound and a corresponding clinical and electrophysiological damage is still under discussion [37]. In other diseases such as entrapment syndromes or pressure palsies, the morphological change often correlates with the function [38]. For example, in cases of acute pressure palsy of radial nerve, conduction blocks can often be found at exactly that section of the upper arm where sonomorphologically focal CSA enlargement occurs [39]. For CSA enlargement in CIDP, only some authors have described similar connections [37, 40]. It was suggested that inflammatory morphological changes in CIDP can probably be displayed by ultrasound even before functional and electrophysiological impairment.

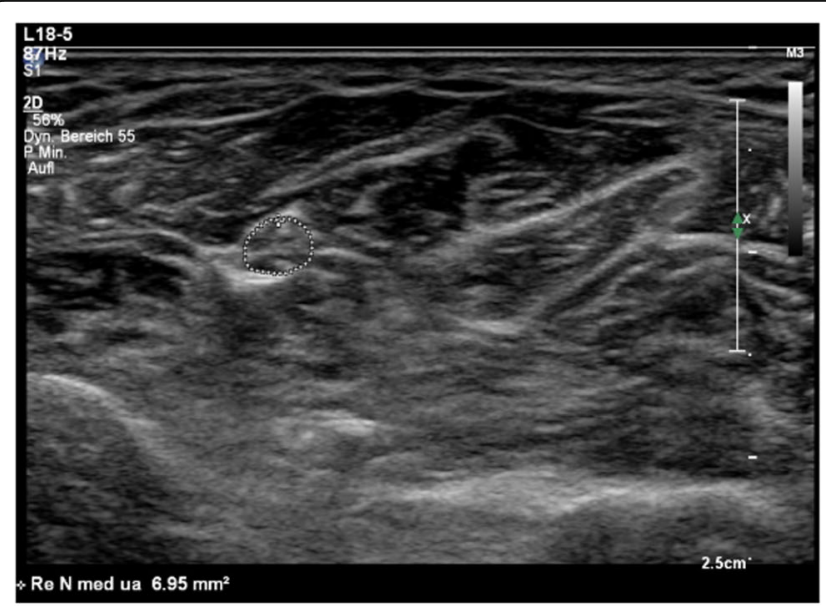

A

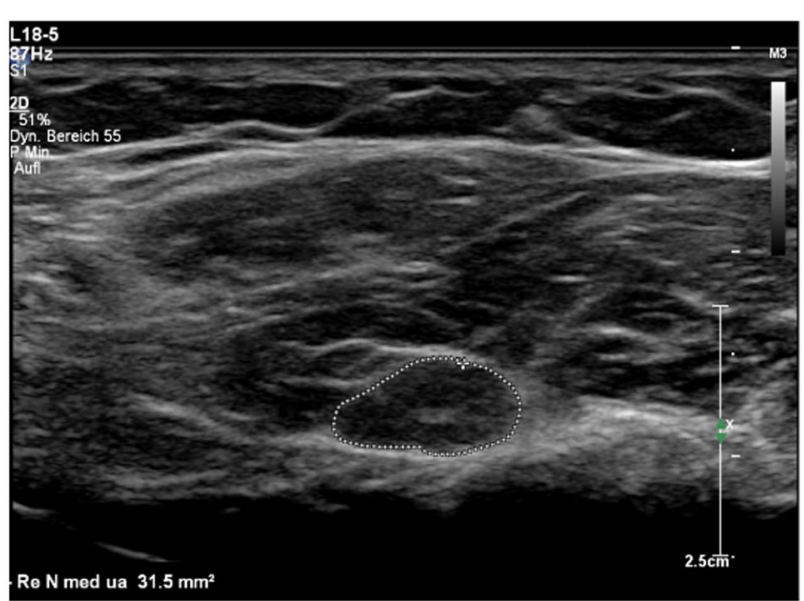

B

Fig. 2 a Normal median nerve at the middle of the forearm between the flexor digitorum profundus and superficialis muscles with a normal CSA of $6.95 \mathrm{~mm}^{2}$ and normal fascicular structure. b Significantly enlarged median nerve at the forearm with a CSA of $31.5 \mathrm{~mm}^{2}$ in a patient with CIDP. Some swollen fascicles and a hypoechoic structure can be depicted. Ultrasound stetting except focus are the same in both images 
In disease course, increase or decrease of CSA enlargement, i.e. measured by intra-nerve CSA variability, can provide information about disease activity and response to therapy $[23,41]$.

Regarding evaluation of echogenicity, HRUS might be useful as prognostic tool, as it was shown that patients with hyperechogenic nerves have a worse prognosis than that with hypoechogenic nerves. Also, the extent of hypoechogenic fraction often occurring along with CSA enlargement correlates to disease course [31, 42].

A limitation of nerve ultrasound is that proximal and deep nerves such as lumbosacral plexus cannot be displayed and that the quality of imaging is dependent on the expertise and experience of the examiner.

\section{Muscle ultrasound}

Muscle ultrasound in CIDP was described to be useful to detect secondary axonal damage via reduced muscle thickness and hyperechogenic remodeling of muscles in one CIDP study [43]. In conditions like motor neuron diseases, muscle ultrasound can also be used to detect fasciculations [44-46], even better than electromyography [47].

\section{Corneal confocal microscopy}

Corneal confocal microscopy (CCM) is a novel promising tool for evaluation of disease activity in CIDP. As a transparent medium, the cornea allows nerves of the subbasal plexus of the ophthalmic branch of the trigeminal nerve and immune cells to be visualized in vivo $[48,49]$ and opens up possibilities for the detection of nerve fiber reduction (Fig. 3). For inflammatory-demyelinating diseases such as CIDP, there are contradictory results about the diagnostic value of the nerve fiber length, density and branching shown by CCM [50, 51]. It is currently still unclear whether these parameters change dynamically in the course of the disease. Moreover, corneal nerve fibers are surrounded by immune cell populations, which can easily be quantified and change dynamically during disease course and might correlate with disease activity [28].

\section{Conclusion of the novel strategies in diagnosis and monitoring: what could be improved based on current knowledge?}

The EFNS/PNS criteria were developed for use in daily clinical care as well as in clinical trials. Nevertheless, they are too complicated for routine use in non-specialized centers and misdiagnosis of CIDP is a current problem. MRI is the only imaging technique mentioned as supportive criterion in EFNS/PNS criteria that involves a high level of technical effort. In contrast, ultrasound is widely available, relatively easy to learn and efficient but is not yet included in the criteria. Nerve imaging offers the possibility of directly imaging inflammation. Nerve ultrasound should play a greater role in diagnosis of CIDP in the future as part of the diagnostic criteria, complementary to electrophysiology and MRI. As monitoring tools nerve conduction studies have shortcomings and the relevance of electromyography is not examined sufficiently. Neuromuscular ultrasound and CCM are promising approaches for disease monitoring, but the number of studies is too small to generally recommend them as standard diagnostics and monitoring.

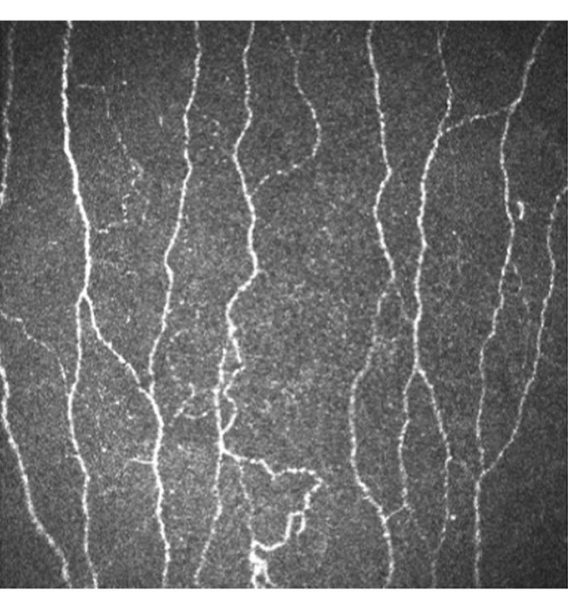

A

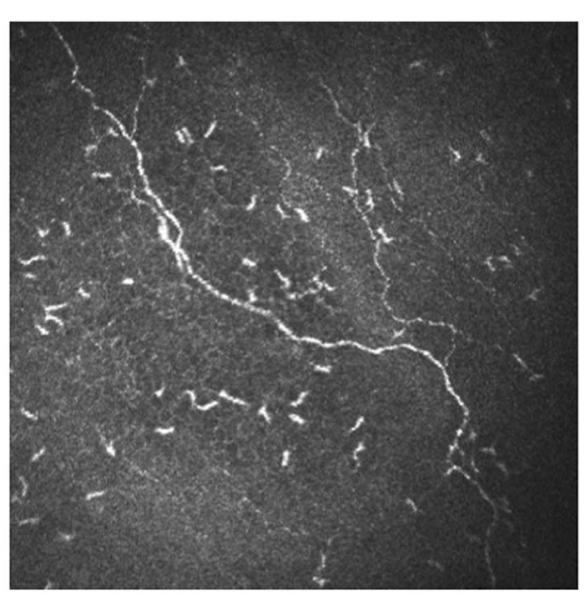

B

Fig. 3 Corneal confocal microscopy showing nerve fiber reduction and immune cell infiltration in a CIDP patient with progressive disease course (b) compared to a healthy person (a). (With the kind permission of Professor Martin Tegenthoff, Bochum, and Dr. Dietrich Sturm, Wuppertal, 2020) 
Hence, there is currently no solitary technical method that can reliably track the course of the disease.

\section{The challenge of understanding nerve inflammation in CIDP}

The clinical heterogeneity, different diagnostic patterns and different treatment response suggest distinct immunological pathophysiology in CIN, CIDP and subgroups. Humoral and cellular, T-cell driven, autoantibody-induced as well as complement-mediated autoimmunity occurs in all CIN and synergistically lead to damage of peripheral nerves [52].

A crucial step is the break-down of the blood-nerve barrier, indicated by increased protein in CSF, gadolinium enhancement in of the nerve trunks, roots and plexuses in MRI studies as well as nerve swelling in ultrasound studies [52-54]. Activated $\mathrm{CD}_{4}^{+} \mathrm{T}$-cells play a major role here, as they are the first cells to cross the blood-nerve barrier. The migrating T-cells secrete cytokines and chemokines and enable macrophages and antibodies to enter the peripheral nervous system. Different $\mathrm{CD}_{4}{ }^{+}$T-cell subsets (Th1, Th17) were described in CIDP subgroups, which indicate differences in underlying T-cell responses between atypical and typical CIDP [55]. Sural nerve biopsies show $\mathrm{CD} 4^{+}, \mathrm{CD}^{+}$cells and macrophages [56-59], but also immunoglobulin and complement on the outer surface of Schwann cells and the compact myelin $[60,61]$.

The concept of classical macrophage-induced myelin destruction, reduced conduction velocities and conduction block resulting from segmental demyelination is considered typical of CIDP and related disorders [62, 63].

Indeed, macrophages are the key player in this concept. They appear as antigen-presenting cells, create a pro-inflammatory environment, destroy the myelin through phagocytosis and directly attack the myelin [64, $65]$. This results in early secondary axonal damage in CIDP [66, 67].

Nerve biopsy shows features of segmental demyelination and remyelination, onion bulb formation (as a result of repeated thin-regenerating and demyelinating Schwann cell effort), nerve edema and occasionally $\mathrm{T}$ cells [68]. Inflammatory infiltrates are typically both endoneurial and epineurial and frequently perivascular [69]. Macrophages are scattered either throughout the endoneurium or in small perivascular clusters in the endoneurium and mediate demyelination [69-71]. Demyelination typically occurs paranodally and only for a short time, Schwann cells quickly remyelinate the destroyed segment insufficiently with shorter internodes and thinner myelin resulting in the onion bulb formation. Schwann cells upregulate the antigen presenting major histocompatibility complex class (MHC)-II, so that a pro-inflammatory environment is maintained in the nerve.

Increased systemic concentrations of TNF $\alpha$ and Interleukin-2 are markers for T-cell activation [72, 73]. However, a single triggering antigen has not yet been found. Therefore, a strong evidence for a molecular mimicry-like mechanism as presumed in GBS is absent. Antibody responses against the myelin proteins P0 and P2 [74] and peripheral myelin protein 22 (PMP22) have been reported $[75,76]$ but are under discussion $[77,78]$. The detection of T cells with $\gamma \delta$-receptors in nerve samples of CIDP underlines the possible pathogenetic role of cellular immune response against non-protein antigens like gangliosides [79].

There is increasing evidence that MHC-I-restricted, $\mathrm{CD}^{+} \mathrm{T}$-cell-mediated attack against peripheral nerve tissue components contributes to the pathogenesis of CIDP [56, 80, 81]. The role of $\mathrm{CD}^{+}{ }^{+}$T-cells is controversial. Similar clonal expansion of $\mathrm{CD}^{+}$cells in sural nerve biopsies and peripheral blood was described [56]. Also, the analysis of the T-cell repertoire in peripheral blood of CIDP patients showed an extensive oligoclonal expansion in $\mathrm{CD}^{+} \mathrm{T}$-cells that was reduced after treatment with intravenous immunoglobulins (IVIg) [82]. An increase of natural killer-T-cells and $\mathrm{CD}^{+} \mathrm{T}$-cells in CSF of CIDP patients was shown recently [81]. These studies suggest a central role of cytotoxic cell types in inflammatory neuropathies. However, up to now, no foreign- or self-antigen has been identified as a $\mathrm{CD}^{+}$target in CIDP. Also, in experimental autoimmune neuritis (EAN, animal model of CIDP), $\mathrm{CD}^{+}{ }^{+}$-cells do not play a significant role [52]. However, EAN may represent human CIDP insufficiently and animal models driven by $\mathrm{CD}^{+} \mathrm{T}$-cells have to be developed. Also, it is currently unknown whether $\mathrm{CD} 8^{+} \mathrm{T}$-cells mediated pathophysiology is part of classical macrophage-induced myelin destruction or a distinct entity.

The role of B-cells is incompletely understood. In spontaneous autoimmune polyneuropathy (SAP) in nonobese diabetic mice, depletion of B-cells and plasmablasts with anti-CD19 antibodies leads to the prevention and attenuation of SAP [83]. The role of regulatory Bcells was described as secondary in this model [84]. In contrast, in immune cell profiling of CSF in CIDP a Bcell pattern was not found [81].

Yet, therapy response after using rituximab as a CD20depleting antibody in CIDP patients may imply a relevant role of B-cells unless their antigen-presenting capacity toward T-lymphocytes stands in the center.

The efficacy of plasma exchange [85] and bortezomib depleting plasma cells [86] in CIDP indicates a pivotal role of humoral mechanisms. Also, immunoglobulin and complement were found deposited on the outer surface of Schwann cells and the compact myelin in sural nerve biopsies $[60,61]$. They were also detected in sera and 
CSF from CIDP patients [87, 88]. Activation of complement system is considered as a relevant part of CIDP pathophysiology [65].

In the context of macrophage-induced demyelination an antibody-mediated pathway is assumed [65]. Autoantibodies from the serum of CIDP patients directed against various myelin proteins such as P0, P2, PMP22 were reported to trigger demyelination after passive transfer in the animal model [52, 87]. However, other authors did not confirm these findings $[6,75,89]$. Therefore, the role of autoantibodies directed against compact myelin proteins in CIDP is still unclear [90].

In contrast, the identification of antibodies against nodal and paranodal structures brought significant progress in understanding about the role of autoantibodies in immune neuropathies [91-93]. The nodal antibodies target antigens such as the nodal proteins neurofascin (NF) 186 and gliomedin or paranodal proteins as Contactin-associated protein (CASPR) 1, NF 155 and contactin 1. These proteins are important in clustering $\mathrm{Na}^{+}$-channels and to maintain the functional structure ("compartmentalization") of the myelinated axon essential for the saltatory conduction. The paranodal disarrangement resulting from the attachment of IgG4 at paranodal junctions and the absence of macrophageinduced demyelination are characteristic pathologic features in patients who have these antibodies [94, 95]. Antibodies against NF 155 and contactin 1 are of IgG4 class, which are not complement-activating [65]. These mechanisms are different to demyelination in classical macrophage-induced CIDP, which is why paranodopathies should be regarded as a distinct disease entity.

The incidence of these paranodal antibodies is reported around 2-13\% in CIDP patients [52, 91, 92]. Some authors recommended the term "seropositive CIDP" for these patients [96]. Patients are clinically characterized as younger than typical CIDP, with a subacute and more severe onset, disabling tremor, ataxia, distal dominant weakness, and poor response to IVIg [96]. It is not known why these (para-) nodal proteins become an autoimmune target. Moreover, unknown nodal autoantibodies are reported in up to $30 \%$ of CIDP patients [97], showing the need for differentiation of further antibodies.

\section{The challenge of understanding aetiology of CIDP Nutrition and environmental factors}

The data on the influence of nutrition and environmental factors in CIDP are scarce. Several studies have shown an association of diet and environmental factors like tobacco and alcohol consumption with the progression of disability in different autoimmune diseases [98, 99]. In neuroinflammatory and neurodegenerative diseases, the immunological influence of the gut microbiome has increasingly gotten into focus of research
[100]. Short-chain fatty acids are reduced in autoimmune diseases as a consequence of an altered gut microbiome. This leads to reduced regulatory $\mathrm{T}$-cell function. Supplementation of short-chain fatty acids like propionic acid shows beneficial immunomodulatory effects, as well as a neuroprotective effect in multiple sclerosis [101, 102]. So far for diseases of the peripheral nervous system short-chain fatty acids were only described in a single case report [103].

Capsaicin is an alkaloid contained in spicy food. It is a direct agonist of the transient receptor potential channel vanilloid subfamily member 1 , that is expressed in different cell types of the nervous system as well as the immune system. This receptor and its modification by capsaicin shows beneficial immunomodulatory effects in EAN [104] as well as in Schwann cells [105].

In real life the influence of these factors is under discussion. In a study with 323 CIDP patients physical activity improves symptom severity, disability and quality of life, but other environmental factors as smoking, alcohol and different dietary regimes did not have an impact on the severity and health perception of CIDP [98].

\section{Genetics}

The association between genetic risk factors and CIDP is under discussion and not yet clearly described. There have been many attempts to find a human leukocyte antigen (HLA) association with CIDP with little success [106]. Some genes show association with disease severity rather than disease susceptibility [106]. The greatest problem is the limited numbers of subjects in the genome association studies. Therefore, it would be beneficial to repeat the studies with larger cohorts [106] as there is need for more detailed molecular studies about HLA influence and modern genetic approaches to CIDP.

\section{The challenge to find potential biomarkers}

Serum neurofilament light chain (sNfL) levels are increased in about a third of CIDP patients and seem to reflect ongoing axonal damage in the peripheral nervous system. sNfL could therefore be a potential biomarker of disease activity however further studies are needed [107-109].

Compound scores created from multi-dimensional CSF parameters like immune cell subtypes could become potential novel diagnostic tools as they differentiate distinct disease mechanisms in subgroups of inflammatory neuropathies [81].

\section{What can we learn from current knowledge of pathophysiology, aetiology and biomarkers for clinical routine?}

The pathophysiology of CIDP shows complex inflammatory mechanisms that result not only in demyelination 
but also in early axonal damage. Therefore, clinical classifications without regarding pathophysiology cannot be comprehensive. The discovery of nodo- and paranodopathies in the recent years illustrates how knowledge of distinct pathophysiology can lead to clinical differentiation of new disease entities. Therefore, biomaterial like blood, CSF and neural tissue of patients with atypical or unusual clinical characteristics needs to be available to the scientific community for further research.

This further understanding of the pathophysiology is crucial to enable specific treatment options.

\section{Treatment of CIDP}

First line treatment options: immunoglobulins, corticosteroids (STE) and plasma exchange (PE)

Immunoglobulins In ICE trial, the largest and longest randomized-controlled trial comparing intravenous immunoglobulin (IVIg) treatment to placebo in 117 patients for 24 weeks, IVIg were applied with an initial dosage of 2 $\mathrm{g} / \mathrm{kg}$ body weight for $2-4$ days, maintained by $1 \mathrm{~g} / \mathrm{kg}$ body weight for 1-2 days every 3 weeks [110], with a therapy response of $54 \%$. Dose reduction after stabilization is attempted individually in everyday clinical practice. Alternatively, subcutaneous application of immunoglobulins (SCIg) in a dosage of $0.2-0.4 \mathrm{~g} / \mathrm{kg}$ per week with individual dosing after stabilization was shown to be effective in 172 patients compared to placebo. $65-81 \%$ of patients treated with SCIg were relapse free in this study [111]. Subcutaneous administration of immunoglobulins is a novel effective option for CIDP patients, enabling convenient treatment at home, reducing infusion-related sideeffects and outpatient visits for CIDP patients.

Mechanism of action of immunoglobulins is multimodal. IgG as the major component of IVIg are considered responsible for most of the immune-modulating effects [112, 113]. Antigen-binding fragment (Fab)dependent effects like blockade of cell-cell interactions and neutralization of cytokines, activated complement proteins and autoantibodies, as well as fragment crystallizable region $(\mathrm{Fc})$-dependent pathways like competitive blockade of low-affinity Fc-Receptors, modulation of Fc-Receptor expression, and saturation of the FcReceptors have been described [114, 115]. These effects ultimately result in a modification of inflammatory mediators, downstream signaling molecules of inflammatory cascades and changes in activation of immune cells $[113,114]$. Difference of immunoglobulin levels in serum before and after IVIg treatment is considered as possible marker for therapy response, as CIDP patients with low post-treatment IgG levels resulting from fast decay of IVIg are associated with clinical worsening during IVIg treatment [116].
Corticosteroids Regarding STE treatment a Cochrane Review from 2017 revealed low evidence levels for treatment with oral or intravenous STE, mostly based on observational, but not on randomized controlled studies [117]. Nevertheless, corticosteroids are commonly used in practice. In a retrospective trial, response rate to STE treatment was around 60\% [118]. Recommended treatment regimens are prednisolone $1 \mathrm{mg} / \mathrm{kg}$ per day orally, or intermittent high dose therapy with $500-1000 \mathrm{mg}$ methylprednisolone for 3-5 days.

Plasma exchange For PE, several available small trials have shown that between 33 and 66\% of CIDP patients have significant short-term improvement from PE, but rapid deterioration may occur after completion of treatment [85]. Reliable data on long-term effects of PE are not available. Usually 5-10 cycles of PE are applied during 2-4 weeks. Immunoadsorption was reported as equally effective as PE [119].

First-line therapies are non-specific and which of these first line therapies is suited best for which patient is still not understood. Future challenge is to differentiate further subgroups to enable more specific therapies.

Other immunotherapies For CIDP patients who do not respond to first line therapies treatment is challenging due to lack of studies. About $25 \%$ of patients do not respond to any of the three first-line therapies $[86,120]$. Further treatment options for these patients include immunosuppressants or even an autologous stem cell transplantion. However, sufficient data on therapy response from controlled trials do not exist and therapies can have severe side effects [121-123]. Randomized studies are only available for azathioprine [124], interferon beta-1a [124-126], fingolimod [127] and methotrexate [128] showing no significant treatment response, but these studies were not large enough to detect minor or moderate benefit [121].

Observational studies including cyclophosphamide, ciclosporin, mycophenolate, rituximab, bortezomib as proteasome antagonist and peripheral blood stem cell transplantation have been performed with positive results, but do not provide enough high-level evidence [121]. Two commonly used drugs are rituximab and cyclophosphamide. Sixty CIDP cases with a response rate of $78 \%$ to rituximab have been reported in different case series [121, 129-132]. For cyclophosphamide fifty-one CIDP cases have been reported, of which 35 (69\%) benefited from therapy [121]. A novel increasingly used treatment in refractory CIDP is bortezomib, a proteasome inhibitor for which efficacy was described in ten CIDP patients [86].

The knowledge about distinct entities of CIN like PDN or nodo- and paranodopathies led to more specific therapies in these diseases: 
PDN are defined as demyelinating neuropathy, clinically often with DADS phenotype, and proof of IgMparaproteinemia with or without anti-MAG-antibodies [133]. PDN is known to respond poorly to standard CIDP therapy with IVIg, STE or PE. Also, effect of other immunosuppressants is limited [133, 134]. Some observational and small controlled studies with cumulatively over 200 PDN patients suggest that rituximab can be helpful in $30-50 \%$ of these patients [134, 135], probably by suppressing IgM and anti-MAG antibody production, however, large randomized controlled studies are lacking.
There are no prospective data about treatment of nodo- and paraponopathies, but observational studies show low therapy response to IVIg [136, 137]. A possible cause of poor IVIg response is that autoantibodies are often IgG4 subgroup, which is not complement activating $[96,137]$. Good response to rituximab was reported in small case series [96, 138, 139].

Drug free treatment options There is evidence from some studies with CIDP patients showing the effect of strength training and physiotherapy to improve muscle strength [140]. Further approaches to improve the

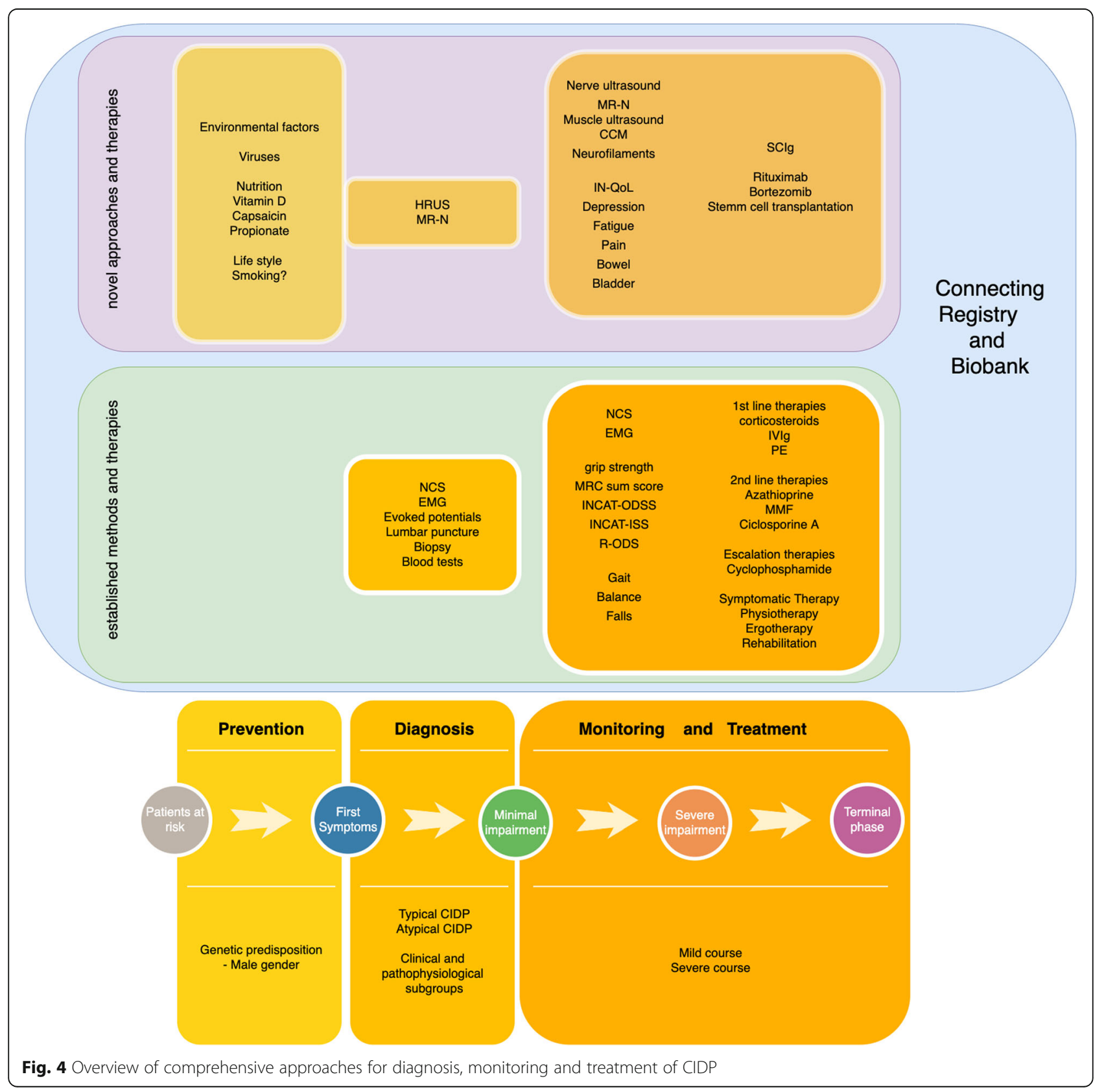


quality of life are neurostimulation or electrostimulation, although no controlled studies are available on this in CIDP, but only in other polyneuropathies and central diseases [141, 142].

\section{What can we conclude about the treatment of CIDP?}

The first line therapy of CIDP is immunologically unspecific. In more than $20 \%$ of the patients these first-line fails. If a patient does not respond to first-line treatment reevaluation of the diagnosis is mandatory. The variable treatment response of different subtypes underlines the importance of improving our knowledge on pathophysiology. But even without specific knowledge of the pathophysiology of certain subtypes, the investigation of subgroup-specific treatment responses is needed to improve treatment response rates.

\section{Conclusion}

CIDP and other chronic inflammatory neuropathies have been better understood in recent years. There are new molecular biological findings and innovative approaches to diagnose and monitor inflammatory neuropathies. Nevertheless, the translation of new diagnostic approaches and new subgroups into diagnostic criteria lacks. Moreover, there is a great need for further knowledge about pathophysiology and therapeutic options of 'seronegative' CIDP itself. Further characterization of distinct subgroups i.e. progressive or treatment refractory patients could enable targeted and more individualized therapy. Until now, treatment decisions in atypical and progressive CIDP are not evidence-based due to the lack of large controlled studies. We hope that the era of individualized treatment in CIDP will make significant progress.

Previous studies, regardless of whether they investigate pathophysiology, biomarkers, genetic or environmental associations or treatment of CIN, have in common that the high number of patients to achieve significant results is difficult to obtain. A further challenge along with the emerging knowledge in clinical and molecular research is to link all these aspects. Possible reasons for delayed implementation of the different fields in CIN are the high degree of specialization of the individual international working groups and inhomogeneous structure of data.

Future research about CIDP with its subtypes and pathophysiology, as well as their therapy, requires combination of high-quality prospective clinical data with the opportunity to structured collection of biomaterials from large numbers of patients. This can only be achieved through multicenter, multidimensional registers for immune-mediated neuropathies with attached biobank (Fig. 4). A high degree of standardization with standard operation protocols must be obtained for biomaterials and their processing in order to make the results valid and comparable. All clinical characteristics, neuroimaging and molecular biological examinations and a comprehensive collection of biomaterials need to be sampled. The aim is the recognition of specific characteristics of the various subtypes of the disease. In addition to a better understanding of the disease, the development of targeted and individualized therapies will be a result of this work.

\section{Abbreviations \\ CIDP: Chronic inflammatory demyelinating polyradiculoneuropathy; EFNS/ PNS: European Federation of Neurological Societies/Peripheral Nerve Society; MRI: Magnetic resonance imaging; CSF: Cerebrospinal fluid; DADS: Distal acquired demyelinating symmetric neuropathy; MADSAM: Multifocal acquired demyelinating sensory and motor neuropathy; MMN: Multifocal motor neuropathy; PDN: Paraproteinemic demyelinating neuropathies; MAG: Myelin-associated glycoprotein; CIN: Chronic inflammatory neuropathies; MRC: Medical Research Council; GBS: Guillain-Barré-Syndrome; ODSS: INCAT-Overall Disability Sum Score; ISS: INCAT sensory sumscore; R- ODS: Rasch-built Overall Disability Scale; MGUS: Monoclonal gammopathy of unclear significance; STIR: Short tau inversion recovery; MRN: Magnetic resonance neurography; DTI: Diffusion tensor imaging; CSA: Cross-sectional area; CCM: Corneal confocal microscopy; MHC: Major histocompatibility complex class; PMP22: Peripheral myelin protein 22; IVlg: Intravenous immunoglobulins; EAN: Experimental autoimmune neuritis; \\ SAP: Spontaneous autoimmune polyneuropathy; NF: Neurofascin; CASP R: Contactin-associated protein; HLA: Human leukocyte antigen; sNfL: Serum neurofilament light chain; STE: Corticosteroids; PE: Plasma exchange; \\ SClg: Subcutaneous immunoglobulins; Fab: Antigen-binding fragment; Fc: Fragment crystallizable region}

\section{Acknowledgements}

Not applicable (review article).

\section{Authors' contributions}

JM: Literature research, drafting and revising the manuscript. ALF: Literature research, drafting and revising the manuscript. TG: Critical comments during drafting and manuscript revision. MS: Critical comments during drafting and manuscript revision. KP: Critical comments during drafting and manuscript revision. RG: Basic idea, critical comments during drafting and manuscript revision. The author(s) read and approved the final manuscript.

Funding

Not applicable (review article).

Availability of data and materials

Not applicable (review article).

Ethics approval and consent to participate

Not applicable (review article).

Consent for publication

Not applicable (review article).

Competing interests

The authors declare that they have no competing interest.

Received: 13 August 2020 Accepted: 4 September 2020

Published online: 08 December 2020

References

1. Dyck, P. J., O'Brien, P. C., Oviatt, K. F., et al. (1982). Prednisone improves chronic inflammatory demyelinating polyradiculoneuropathy more than no treatment. Annals of Neurology, 11, 136-141.

2. den Bergh, P. Y. K. V., Hadden, R. D. M., Bouche, P., et al. (2010). European Federation of Neurological Societies/peripheral nerve society guideline on management of chronic inflammatory demyelinating polyradiculoneuropathy: Report of a joint task force of the European 
Federation of Neurological Societies and the peripheral nerve society First revision. European Journal of Neurology, 17, 356-363.

3. Mahdi-Rogers, M., \& Hughes, R. A. C. (2013). Epidemiology of chronic inflammatory neuropathies in Southeast England. European Journal of Neurology, 21, 28-33.

4. Martyn, C. N., \& Hughes, R. A. C. (1997). Epidemiology of peripheral neuropathy. Journal of Neurology, Neurosurgery, and Psychiatry, 62, 310-318.

5. McLeod, J. G., Pollard, J. D., Macaskill, P., Mohamed, A., Spring, P., \& Khurana, $\checkmark$. (1999). Prevalence of chronic inflammatory demyelinating polyneuropathy in New South Wales, Australia. Annals of Neurology, 46, 910 913.

6. Kieseier, B. C., Mathey, E. K., Sommer, C., \& Hartung, H.P. (2018). Immunemediated neuropathies. Nature Reviews. Disease Primers, 4, 31.

7. Doneddu, P. E., Bianchi, E., Cocito, D., et al. (2019). Risk factors for chronic inflammatory demyelinating polyradiculoneuropathy (CIDP): Antecedent events, lifestyle and dietary habits. Data from the Italian CIDP database. European Journal of Neurology, 27, 136-143.

8. Breiner, A., \& Brannagan, T. H. (2013). Comparison of sensitivity and specificity among 15 criteria for chronic inflammatory demyelinating polyneuropathy. Muscle \& Nerve, 50, 40-46.

9. Allen, J. A. (2020). The misdiagnosis of CIDP: A review. Neurology and Therapy, 9, 43-54.

10. Breiner, A., Bourque, P. R., \& Allen, J. A. (2019). Updated cerebrospinal fluid total protein reference values improve chronic inflammatory demyelinating polyneuropathy diagnosis. Muscle \& Nerve, 60, 180-183.

11. Group TFCS (2008). Recommendations on diagnostic strategies for chronic inflammatory demyelinating polyradiculoneuropathy. Postgraduate Medical Journal, 84, 378.

12. Vallat, J.-M., Tabaraud, F., Magy, L., et al. (2003). Diagnostic value of nerve biopsy for atypical chronic inflammatory demyelinating polyneuropathy: Evaluation of eight cases. Muscle \& Nerve, 27, 478-485.

13. Boukhris, S., Magy, L., Kabore, R., et al. (2004). Atypical electrophysiologic findings in chronic inflammatory demyelinating polyneuropathy (CIDP) Diagnosis confirmed by nerve biopsy. Neurophysiologie Clinique/Clinical Neurophysiology, 34, 71-79.

14. Devic, P., Petiot, P., \& Mauguiere, F. (2016). Diagnostic utility of somatosensory evoked potentials in chronic polyradiculopathy without electrodiagnostic signs of peripheral demyelination. Muscle \& Nerve, 53, 7883.

15. Doneddu, P. E., Cocito, D., Manganelli, F., et al. (2018). Atypical CIDP: Diagnostic criteria, progression and treatment response. Data from the Italian CIDP database. Journal of Neurology, Neurosurgery, and Psychiatry, 90, 125-132.

16. Aids to the examination of the peripheral nervous system - MRC Memorandum No.45 (superseding War Memorandum No.7).pdf. Epub n.d. https://mrc.ukri.org/research/facilities-and-resources-for-researchers/mrcscales/mrc-muscle-scale. Accessed 08 Sept 2020.

17. Merkies, I. S. J., Schmitz, P. I. M., van der Meché, F. G. A., Samijn, J. P. A., \& van Doorn, P. A. (2002). Clinimetric evaluation of a new overall disability scale in immune mediated polyneuropathies. Journal of Neurology, Neurosurgery, and Psychiatry, 72, 596.

18. Merkies, I. S., Schmitz, P. I., van der Meché, F. G., \& Doorn, P. A. V. (2000). Psychometric evaluation of a new sensory scale in immune-mediated polyneuropathies. Inflammatory neuropathy cause and treatment (INCAT) group. Neurology., 54, 943-949.

19. Vanhoutte, E. K., Draak, T. H. P., Gorson, K. C., et al. (2015). Impairment measures versus inflammatory RODS in GBS and CIDP: A responsiveness comparison. Journal of the Peripheral Nervous System, 20, 289-295.

20. van Nes, S. I., Vanhoutte, E. K., van Doorn, P. A., et al. (2011). Rasch-built overall disability scale (R-ODS) for immune-mediated peripheral neuropathies. Neurology., 76, 337-345.

21. Draak, T. H. P., Faber, C. G., Merkies, I. S. J., \& Group P study (2017). Quality of life in inflammatory neuropathies: The IN-QoL. Journal of Neurology, Neurosurgery, and Psychiatry, 89, 256-262.

22. Vanhoutte, E. K., Latov, N., Deng, C., et al. (2013). Vigorimeter grip strength in CIDP: A responsive tool that rapidly measures the effect of IVIG - The ICE study. European Journal of Neurology, 20, 748-755.

23. Fisse, A. L., Pitarokoili, K., Trampe, N., et al. (2018). Clinical, Sonographic, and Electrophysiologic longitudinal features of chronic inflammatory demyelinating polyneuropathy. Journal of Neuroimaging, 9, 402.
24. Décard, B. F., Pham, M., \& Grimm, A. (2018). Ultrasound and MRI of nerves for monitoring disease activity and treatment effects in chronic dysimmune neuropathies - current concepts and future directions. Clinical Neurophysiology, 129, 155-167.

25. Kronlage, M., Pitarokoili, K., Schwarz, D., et al. (2017). Diffusion tensor imaging in chronic inflammatory demyelinating polyneuropathy: Diagnostic accuracy and correlation with electrophysiology. Investigative Radiology, 52, 701-707.

26. Lichtenstein, T., Sprenger, A., Weiss, K., et al. (2017). MRI biomarkers of proximal nerve injury in CIDP. Annals of Clinical Translational Neurology, 5, 19-28.

27. Pitarokoili, K., Schlamann, M., Kerasnoudis, A., Gold, R., \& Yoon, M. S. (2015). Comparison of clinical, electrophysiological, sonographic and MRI features in CIDP. Journal of the Neurological Sciences, 357, 198-203.

28. Pitarokoili, K., Sturm, D., Labedi, A., et al. (2019). Neuroimaging markers of clinical progression in chronic inflammatory demyelinating polyradiculoneuropathy. Therapeutic Advances in Neurological Disorders, 12 1756286419855485

29. Grimm, A., Winter, N., Rattay, T. W., et al. (2017). A look inside the nerve morphology of nerve fascicles in healthy controls and patients with polyneuropathy. Clinical Neurophysiology, 128, 2521-2526.

30. Padua, L., Granata, G., Sabatelli, M., et al. (2014). Heterogeneity of root and nerve ultrasound pattern in CIDP patients. Clinical Neurophysiology, 125, 160-165.

31. Fisse, A. L., Pitarokoili, K., Motte, J., et al. (2019). Nerve echogenicity and intranerve CSA variability in high-resolution nerve ultrasound (HRUS) in chronic inflammatory demyelinating polyneuropathy (CIDP). Journal of Neurology, 266, 468-475.

32. Grimm, A., Décard, B. F., Axer, H., \& Fuhr, P. (2015). The ultrasound pattern sum score - UPSS. A new method to differentiate acute and subacute neuropathies using ultrasound of the peripheral nerves. Clinical Neurophysiology, 126, 2216-2225.

33. Kerasnoudis, A., Pitarokoili, K., Gold, R., \& Yoon, M.-S. (2015). Bochum ultrasound score allows distinction of chronic inflammatory from multifocal acquired demyelinating polyneuropathies. Journal of the Neurological Sciences, 348, 211-215.

34. Goedee, H. S., van der Pol, W. L., van Asseldonk, J.-T. H., et al. (2017) Diagnostic value of sonography in treatment-naive chronic inflammatory neuropathies. Neurology., 88, 143-151.

35. Zaidman, C. M., \& Pestronk, A. (2014). Nerve size in chronic inflammatory demyelinating neuropathy varies with disease activity and therapy response over time: A retrospective ultrasound study. Muscle \& Nerve, 50, 733-738.

36. Grimm, A., Rattay, T. W., Winter, N., \& Axer, H. (2016). Peripheral nerve ultrasound scoring systems: Benchmarking and comparative analysis. Journal of Neurology, 264, 1-11.

37. Kerasnoudis, A., Pitarokoili, K., Behrendt, V., Gold, R., \& Yoon, M. S. (2014). Correlation of nerve ultrasound, electrophysiological and clinical findings in chronic inflammatory demyelinating polyneuropathy. Journal of Neuroimaging, 25, 207-216.

38. Cartwright, M. S., \& Walker, F. O. (2013). Neuromuscular ultrasound in common entrapment neuropathies. Muscle \& Nerve, 48, 696-704.

39. Motte, J., Gold, R., \& Krogias, C. (2018). Monitoring of structural changes in the course of acute compressive radial neuropathies by means of high resolution nerve ultrasound. Journal of the Neurological Sciences, 391, 45-47.

40. Scheidl, E., Böhm, J., Simó, M., et al. (2012). Ultrasonography of MADSAM neuropathy: Focal nerve enlargements at sites of existing and resolved conduction blocks. Neuromuscular Disorders, 22, 627-631.

41. Allen, J. A., Merkies, I. S. J., \& Lewis, R. A. (2020). Monitoring clinical course and treatment response in chronic inflammatory demyelinating polyneuropathy during routine care: a review of clinical and laboratory assessment measures. JAMA neurology, 77. Advance online publication. https://doi.org/10.1001/jamaneurol.2020.0781.

42. Gamber, D., Motte, J., Kerasnoudis, A., et al. (2020). High-resolution nerve ultrasound to assess nerve echogenicity, fascicular count, and crosssectional area using Semiautomated analysis. Journal of Neuroimaging, 30, 493-502.

43. Hokkoku, K., Matsukura, K., Uchida, Y., et al. (2017). Quantitative muscle ultrasound is useful for evaluating secondary axonal degeneration in chronic inflammatory demyelinating polyneuropathy. Brain and Behavior: A Cognitive Neuroscience Perspective, 7, e00812. 
44. Pillen, S., Boon, A., \& van Alfen, N. (2016). Muscle ultrasound. Handbook of Clinical Neurology, 136, 843-853.

45. Karvelas, K. R., Hommel, A. L., Cartwright, M. S., Walker, F. O., \& HobsonWebb, L. D. (2018). Sonographic similarities of inclusion body myositis and Myotonic dystrophy. Muscle \& Nerve, 58, E25-E26.

46. Heckmatt, J. Z., Leeman, S., \& Dubowitz, V. (1982). Ultrasound imaging in the diagnosis of muscle disease. Journal of Pediatrics, 101, 656-660.

47. Duarte, M. L., lared, W., Oliveira, A. S. B., Santos, L. R. D., \& Peccin, M. S. (2020). Ultrasound versus electromyography for the detection of fasciculation in amyotrophic lateral sclerosis: Systematic review and metaanalysis. Radiologia Brasileira, 53, 116-121.

48. Ferrari, G., Nalassamy, N., Downs, H., Dana, R., \& Oaklander, A. L. (2013). Corneal innervation as a window to peripheral neuropathies. Experimental Eye Research, 113, 148-150.

49. Hertz, P., Bril, V., Orszag, A., et al. (2011). Reproducibility of in vivo corneal confocal microscopy as a novel screening test for early diabetic sensorimotor polyneuropathy. Diabetic Medicine, 28, 1253-1260.

50. Stettner, M., Hinrichs, L., Guthoff, R., et al. (2016). Corneal confocal microscopy in chronic inflammatory demyelinating polyneuropathy. Annals of Clinical Translational Neurology, 3, 88-100.

51. Schneider, C., Bucher, F., Cursiefen, C., Fink, G. R., Heindl, L. M., \& Lehmann, H. C. (2014). Corneal confocal microscopy detects small fiber damage in chronic inflammatory demyelinating polyneuropathy (CIDP). Journal of the Peripheral Nervous System, 19, 322-327.

52. Mathey, E. K., Park, S. B., Hughes, R. A. C., et al. (2015). Chronic inflammatory demyelinating polyradiculoneuropathy: From pathology to phenotype. Journal of Neurology, Neurosurgery, and Psychiatry, 86, 973-985.

53. Kuwabara, S., Nakajima, M., Matsuda, S., \& Hattori, T. (1997). Magnetic resonance imaging at the demyelinative foci in chronic inflammatory demyelinating polyneuropathy. Neurology., 48, 874-877.

54. Lehmann, H. C., Burke, D., \& Kuwabara, S. (2019). Chronic inflammatory demyelinating polyneuropathy: Update on diagnosis, immunopathogenesis and treatment. Journal of Neurology, Neurosurgery, and Psychiatry, 90, 981987.

55. Klehmet, J., Staudt, M., Diederich, J.-M., et al. (2017). Neurofascin (NF)155and NF186-specific T cell response in a patient developing a central Pontocerebellar demyelination after 10 years of CIDP. Frontiers in Neurology, 8, 347-344.

56. Schneider-Hohendorf, T., Schwab, N., Uçeyler, N., Gobel, K., Sommer, C., \& Wiendl, H. (2012). CD8+ T-cell immunity in chronic inflammatory demyelinating polyradiculoneuropathy. Neurology, 78, 402-408.

57. Kiefer, R., Kieseier, B. C., Brück, W., Hartung, H. P., \& Toyka, K. V. (1998). Macrophage differentiation antigens in acute and chronic autoimmune polyneuropathies [published erratum appears in brain 1998 Jun;121(Pt 6): 1190]. Brain., 121, 469-479.

58. Schmidt, B., Toyka, K. V., Kiefer, R., Full, J., Hartung, H.P., \& Pollard, J. (1996), Inflammatory infiltrates in sural nerve biopsies in Guillain-Barré syndrome and chronic inflammatory demyelinating neuropathy. Muscle \& Nerve, 19, 474-487.

59. Cornblath, D. R., Griffin, D. E., Welch, D., Griffin, J. W., \& McArthur, J. C. (1990). Quantitative analysis of endoneurial T-cells in human sural nerve biopsies. Journal of Neuroimmunology, 26, 113-118.

60. Dalakas, M. C. \& Engel, W. K. (1980). Immunoglobulin and Complement Deposits in Nerves of Patients With Chronic Relapsing Polyneuropathy. Archives of Neurology-Chicago, 37, 637-640.

61. Hays, A. P., Lee, S. S. L., \& Latov, N. (1988). Immune reactive C3d on the surface of myelin sheaths in neuropathy. Journal of Neuroimmunology, 18, 231-244.

62. Dyck, P. J. B., \& Tracy, J. A. (2018). History, diagnosis, and Management of Chronic Inflammatory Demyelinating Polyradiculoneuropathy. Mayo Clinic Proceedings [online serial], 93, 777-793 10.1016/j.mayocp.2018.03.026.

63. Vallat, J.-M., Sommer, C., \& Magy, L. (2010). Chronic inflammatory demyelinating polyradiculoneuropathy: Diagnostic and therapeutic challenges for a treatable condition. Lancet Neurology, 9, 402-412.

64. Kiefer, R., Kieseier, B. C., Stoll, G., \& Hartung, H.P. (2001). The role of macrophages in immune-mediated damage to the peripheral nervous system. Progress in Neurobiology, 64, 109-127.

65. Koike, H., Nishi, R., Ikeda, S., et al. (2018). Ultrastructural mechanisms of macrophage-induced demyelination in CIDP. Neurology., 91, 1051-1060.

66. Toyka, K. V., \& Gold, R. (2003). The pathogenesis of CIDP: Rationale for treatment with immunomodulatory agents. Neurology., 60, S2-S7.
67. Bosboom, W. M. J., van den Berg, L. H., Franssen, H., et al. (2001). Diagnostic value of sural nerve demyelination in chronic inflammatory demyelinating polyneuropathy. Brain., 124, 2427-2438.

68. Rajabally, Y. A., Stettner, M., Kieseier, B. C., Hartung, H.P., \& Malik, R. A. (2017). CIDP and other inflammatory neuropathies in diabetes - Diagnosis and management. Nature Publishing Group, 13, 599-611.

69. Sommer, C., Koch, S., Lammens, M., Gabreels-Festen, A., Stoll, G., \& Toyka, K. V. (2005). Macrophage clustering as a diagnostic marker in sural nerve biopsies of patients with CIDP. Neurology, 65, 1924-1929.

70. Vital, A., Lagueny, A., Julien, J., et al. (2000). Chronic inflammatory demyelinating polyneuropathy associated with dysglobulinemia: a peripheral nerve biopsy study in 18 cases. Acta Neuropathologica, 100, 6368.

71. Vital, C., Vital, A., Lagueny, A., et al. (2009). Chronic inflammatory demyelinating polyneuropathy: Immunopathological and Ultrastructural study of peripheral nerve biopsy in 42 cases. Ultrastructural Pathology, 24, 363-369.

72. Misawa, S., Kuwabara, S., Mori, M., Kawaguchi, N., Yoshiyama, Y., \& Hattori, T. (2001). Serum levels of tumor necrosis factor-alpha in chronic inflammatory demyelinating polyneuropathy. Neurology [online serial]., 56, 666-669 Accessed at: http://www.neurology.org/cgi/doi/10.1212/WNL.56.5.666.

73. Hartung, H. P., Reiners, K., Schmidt, B., Stoll, G., \& Toyka, K. V. (1991). Serum interleukin-2 concentrations in Guillain-Barré syndrome and chronic idiopathic demyelinating polyradiculoneuropathy: Comparison with other neurological diseases of presumed immunopathogenesis. Annals of Neurology, 30, 48-53.

74. Khalili-Shirazi, A., Atkinson, P., Gregson, N., \& Hughes, R. A. C. (1993). Antibody responses to P0 and P2 myelin proteins in Guillain-Barré syndrome and chronic idiopathic demyelinating polyradiculoneuropathy. Journal of Neuroimmunology, 46, 245-251.

75. Sanvito, L., Makowska, A., Mahdi-Rogers, M., et al. (2009). Humoral and cellular immune responses to myelin protein peptides in chronic inflammatory demyelinating polyradiculoneuropathy. Journal of Neurology, Neurosurgery, and Psychiatry, 80, 333-338.

76. Gabriel, C. M., Gregson, N. A., \& Hughes, R. A. C. (2000). Anti-PMP22 antibodies in patients with inflammatory neuropathy. Journal of Neuroimmunology, 104, 139-146.

77. Inglis, H. R., Csurhes, P. A., \& McCombe, P. A. (2006). Antibody responses to peptides of peripheral nerve myelin proteins $\mathrm{P} 0$ and $\mathrm{P} 2$ in patients with inflammatory demyelinating neuropathy. Journal of Neurology, Neurosurgery, and Psychiatry, 78, 419-422.

78. Kwa, M. S. G., Schaik, I. N. V., Brand, A., Baas, F., \& Vermeulen, M. (2001), Investigation of serum response to PMP22, connexin 32 and P0 in inflammatory neuropathies. Journal of Neuroimmunology, 116, 220-225.

79. Winer, J., Hughes, S., Cooper, J., Ben-Smith, A., \& Savage, C. (2002). $ү \delta$ T cells infiltrating sensory nerve biopsies from patients with inflammatory neuropathy. Journal of Neurology, 249, 616-621.

80. Schafflick, D., Kieseier, B. C., Wiendl, H., \& Hörste, G. M. z. (2017). Novel pathomechanisms in inflammatory neuropathies. Journal of Neuroinflammation, 14, 1-17.

81. Heming, M., Schulte-Mecklenbeck, A., Brix, T., et al. (2019). Immune cell profiling of the cerebrospinal fluid provides Pathogenetic insights into inflammatory neuropathies. Frontiers in Immunology, 10, 515.

82. Mausberg, A. K., Dorok, M., Stettner, M., et al. (2013). Recovery of the T-cell repertoire in CIDP by IV immunoglobulins. Neurology, 80, 296-303.

83. Abraham, P. M., Quan, S. H., Dukala, D., \& Soliven, B. (2014). CD19 as a therapeutic target in a spontaneous autoimmune polyneuropathy: CD19 in SAP. Clinical and Experimental Immunology, 175, 181-191.

84. Quan, S., Sheng, J. R., Abraham, P. M., \& Soliven, B. (2016). Regulatory T and B lymphocytes in a spontaneous autoimmune polyneuropathy: T regs and $B$ regs in SAP. Clinical and Experimental Immunology, 184, 50-61.

85. Mehndiratta, M. M., Hughes, R. A., \& Pritchard, J. (2015). Plasma exchange for chronic inflammatory demyelinating polyradiculoneuropathy. Cochrane Database of Systematic Reviews, (8), CD003906. https://doi.org/10.1002/ 14651858.CD003906.pub4.

86. Pitarokoili, K., Yoon, M.-S., Kröger, I., Reinacher-Schick, A., Gold, R., \& Schneider-Gold, C. (2017). Severe refractory CIDP: A case series of 10 patients treated with bortezomib. Journal of Neurology, 264, 2010-2020.

87. Yan, W. X., Taylor, J., Andrias-Kauba, S., \& Pollard, J. D. (2001). Passive transfer of demyelination by serum or IgG from chronic inflammatory demyelinating polyneuropathy patients. Annals of Neurology, 47, 765-775. 
88. Quast, l., Keller, C. W., Hiepe, F., Tackenberg, B., \& Lünemann, J. D. (2016). Terminal complement activation is increased and associated with disease severity in CIDP. Annals of Clinical Translational Neurology, 3, 730-735.

89. Lim, J. P., Devaux, J., \& Yuki, N. (2014). Peripheral nerve proteins as potential autoantigens in acute and chronic inflammatory demyelinating polyneuropathies. Autoimmunity Reviews, 13, 1070-1078.

90. Harvey, G. K., Gold, R., Hartung, H.-P., \& Toyka, K. V. (1995). Non-neuralspecific $T$ lymphocytes can orchestrate inflammatory peripheral neuropathy. Brain., 118, 1263-1272.

91. Querol, L., Devaux, J., Rojas-Garcia, R., \& Illa, I. (2017). Autoantibodies in chronic inflammatory neuropathies: Diagnostic and therapeutic implications. Nature Publishing Group, 13, 533-547.

92. Stathopoulos, P., Alexopoulos, H., \& Dalakas, M. C. (2015). Autoimmune antigenic targets at the node of Ranvier in demyelinating disorders. Nature Reviews. Neurology, 11, 143-156.

93. Delmont, E., Manso, C., Querol, L., et al. (2017). Autoantibodies to nodal isoforms of neurofascin in chronic inflammatory demyelinating polyneuropathy. Brain., 140, 1851-1858.

94. Koike, H., Kadoya, M., Kaida, K.-I., et al. (2017). Paranodal dissection in chronic inflammatory demyelinating polyneuropathy with anti-neurofascin-155 and anti-contactin-1 antibodies. Journal of Neurology, Neurosurgery, and Psychiatry, 88, 465-473.

95. Koike, H., Katsuno, M., \& Sobue, G. (2018). Deciphering the mechanism and spectrum of chronic inflammatory demyelinating polyneuropathy using morphology. Clinical and Experimental Neuroimmunology, 9, 35-46.

96. Vural, A., Doppler, K., \& Meinl, E. (2018). Autoantibodies against the node of Ranvier in seropositive chronic inflammatory demyelinating polyneuropathy: Diagnostic, pathogenic, and therapeutic relevance. Frontiers in Immunology, 9, 1029.

97. Devaux, J. J., Odaka, M., \& Yuki, N. (2012). Nodal proteins are target antigens in Guillain-Barré syndrome. Journal of the Peripheral Nervous System, 17, 6271.

98. Doneddu, P. E., Bianchi, E., Cocito, D., et al. (2020). Impact of environmental factors and physical activity on disability and quality of life in CIDP. Journal of Neurology, 267(9), 2683-2691.

99. Jörg, S., Grohme, D. A., Erzler, M., et al. (2016). Environmental factors in autoimmune diseases and their role in multiple sclerosis. Cellular and Molecular Life Sciences, 73, 4611-4622.

100. Hirschberg, S., Gisevius, B., Duscha, A., \& Haghikia, A. (2019). Implications of diet and the gut microbiome in Neuroinflammatory and neurodegenerative diseases. International Journal of Molecular Sciences, 20, 3109.

101. Haghikia, A., Jörg, S., Duscha, A., et al. (2015). Dietary fatty acids directly impact central nervous system autoimmunity via the small intestine. Immunity., 43, 817-829.

102. Duscha, A., Gisevius, B., Hirschberg, S., et al. (2020). Propionic Acid Shapes the Multiple Sclerosis Disease Course by an Immunomodulatory Mechanism. Cell, 180, 1067-1080.e16.

103. Yoon, M.-S., Pitarokoili, K., Sturm, D., Haghikia, A., Gold, R., \& Fisse, A. L. (2018). Treatment of an acute motor and sensory axonal neuropathy with propionate in a 33-year-old male. Therapeutic Advances in Neurological Disorders, 11, 175628641880958-175628641880956.

104. Motte, J., Ambrosius, B., Grüter, T., et al. (2018). Capsaicin-enriched diet ameliorates autoimmune neuritis in rats. Journal of Neuroinflammation, 15, 122.

105. Grüter, T., Blusch, A., Motte, J., et al. (2020). Immunomodulatory and antioxidative effect of the direct TRPV1 receptor agonist capsaicin on Schwann cells. Journal of Neuroinflammation, 17, 145.

106. Blum, S., \& McCombe, P. A. (2014). Genetics of Guillain-Barré syndrome (GBS) and chronic inflammatory demyelinating polyradiculoneuropathy (CIDP): Current knowledge and future directions. Journal of the Peripheral Nervous System, 19, 88-103

107. Petzold, A., Brettschneider, J., Jin, K., et al. (2009). CSF protein biomarkers for proximal axonal damage improve prognostic accuracy in the acute phase of Guillain-Barré syndrome. Muscle \& Nerve, 40, 42-49.

108. Mariotto, S., Farinazzo, A., Magliozzi, R., Alberti, D., Monaco, S., \& Ferrari, S. (2018). Serum and cerebrospinal neurofilament light chain levels in patients with acquired peripheral neuropathies. Journal of the Peripheral Nervous System Epub.

109. van Lieverloo, G. G. A., Wieske, L., Verhamme, C., et al. (2019). Serum neurofilament light chain in chronic inflammatory demyelinating polyneuropathy. Journal of the Peripheral Nervous System Epub.

110. Hughes, R. A., Donofrio, P., Bril, V., et al. (2008). Intravenous immune globulin ( $10 \%$ caprylate-chromatography purified) for the treatment of chronic inflammatory demyelinating polyradiculoneuropathy (ICE study): a randomised placebo-controlled trial. The Lancet Neurology [online serial], 7 , 136-144 Accessed at: message: \%3Cf720eff71eb4defaabf55dad1ee51e96@ruhr-uni-bochum.de\%3E.

111. van Schaik, I. N., Bril, V., van Geloven, N., et al. (2018). Subcutaneous immunoglobulin for maintenance treatment in chronic inflammatory demyelinating polyneuropathy (PATH): A randomised, double-blind, placebo-controlled, phase 3 trial. Lancet Neurology, 17, 35-46.

112. Kuitwaard, K., de Gelder, J., Tio-Gillen, A. P., et al. (2009). Pharmacokinetics of intravenous immunoglobulin and outcome in Guillain-Barré syndrome. Annals of Neurology, 66, 597-603.

113. Dalakas, M. C. (2002). Mechanisms of action of IVlg and therapeutic considerations in the treatment of acute and chronic demyelinating neuropathies. Neurology., 59, S13-S21.

114. Galeotti, C., Kaveri, S. V., \& Bayry, J. (2017). IVIG-mediated effector functions in autoimmune and inflammatory diseases. International Immunology, 29, 491-498.

115. Lünemann, J. D., Nimmerjahn, F., \& Dalakas, M. C. (2015). Intravenous immunoglobulin in neurology-Mode of action and clinical efficacy. Nature Reviews. Neurology, 11, 80-89.

116. Ritter, C., Bobylev, I., \& Lehmann, H. C. (2015). Chronic inflammatory demyelinating polyneuropathy (CIDP): Change of serum IgG dimer levels during treatment with intravenous immunoglobulins. Journal of Neuroinflammation, 12, 148.

117. Hughes, R. A., Mehndiratta, M. M., \& Rajabally, Y. A. (2017). Corticosteroids for chronic inflammatory demyelinating polyradiculoneuropathy. Cochrane Database of Systematic Reviews Epub.

118. van Lieverloo, G. G. A., Peric, S., Doneddu, P. E., et al. (2018). Corticosteroids in chronic inflammatory demyelinating polyneuropathy. Journal of Neurology, 265, 2052-2059.

119. Lieker, I., Slowinski, T., Harms, L., Hahn, K., \& Klehmet, J. (2017). A prospective study comparing tryptophan immunoadsorption with therapeutic plasma exchange for the treatment of chronic inflammatory demyelinating polyneuropathy*. Journal of Clinical Apheresis, 32, 486493.

120. Mahdi-Rogers, M., van Doorn, P. A., \& Hughes, R. A. C. (2013). Immunomodulatory treatment other than corticosteroids, immunoglobulin and plasma exchange for chronic inflammatory demyelinating polyradiculoneuropathy. Cochrane Database of Systematic Reviews, 35, CD003280 Mahdi-Rogers M, editor.

121. Mahdi-Rogers, M., Brassington, R., Gunn, A. A., van Doorn, P. A., \& Hughes, R. A. (2017). Immunomodulatory treatment other than corticosteroids, immunoglobulin and plasma exchange for chronic inflammatory demyelinating polyradiculoneuropathy. Cochrane Database of Systematic Reviews., 5, CD003280.

122. Mahdi-Rogers, M., Kazmi, M., Ferner, R., et al. (2009). Autologous peripheral blood stem cell transplantation for chronic acquired demyelinating neuropathy. Journal of the Peripheral Nervous System, 14, 118-124.

123. Yoon, M.-S., Chan, A., \& Gold, R. (2011). Standard and escalating treatment of chronic inflammatory demyelinating polyradiculoneuropathy. Therapeutic Advances in Neurological Disorders., 4, 193-200.

124. Dyck, P. J., O'Brien, P., Swanson, C., Low, P., \& Daube, J. (1985). Combined azathioprine and prednisone in chronic inflammatory-demyelinating polyneuropathy. Neurology., 35, 1173-1173.

125. Pritchard, J., Gray, I. A., Idrissova, Z. R., et al. (2003). A randomized controlled trial of recombinant interferon-beta 1a in Guillain-Barre syndrome. Neurology., 61, 1282-1284.

126. Hughes, R. A. C., Gorson, K. C., Cros, D., et al. (2010). Intramuscular interferon beta-1a in chronic inflammatory demyelinating polyradiculoneuropathy. Neurology., 74, 651-657.

127. Hughes, R., Dalakas, M. C., Merkies, l., et al. (2018). Oral fingolimod for chronic inflammatory demyelinating polyradiculoneuropathy (FORCIDP trial): A doubleblind, multicentre, randomised controlled trial. Lancet Neurology, 17, 689-698.

128. Group RT (2009). Randomised controlled trial of methotrexate for chronic inflammatory demyelinating polyradiculoneuropathy (RMC trial): A pilot, multicentre study. Lancet Neurology, 8, 158-164.

129. Muley, S. A., Jacobsen, B., Parry, G., et al. (2020). Rituximab in refractory chronic inflammatory demyelinating polyneuropathy. Muscle \& Nerve Epub.

130. Roux, T., Debs, R., Maisonobe, T., et al. (2018). Rituximab in chronic inflammatory demyelinating polyradiculoneuropathy with associated diseases. Journal of the Peripheral Nervous System, 23, 235-240. 
131. Velardo, D., Riva, N., Carro, U. D., Bianchi, F., Comi, G., \& Fazio, R. (2017). Rituximab in refractory chronic inflammatory demyelinating polyradiculoneuropathy: Report of four cases. Journal of Neurology, 264, 1011-1014.

132. Benedetti, L., Briani, C., Franciotta, D., et al. (2011). Rituximab in patients with chronic inflammatory demyelinating polyradiculoneuropathy: A report of 13 cases and review of the literature. Journal of Neurology, Neurosurgery, and Psychiatry, 82, 306-308.

133. European Federation of Neurological Societies/Peripheral Nerve Society Guideline on management of paraproteinemic demyelinating neuropathies (2006). Report of a joint task force of the European Federation of Neurological Societies and the Peripheral Nerve Society. Journal of the Peripheral Nervous System, 11, 9-19.

134. Dalakas, M. C., Rakocevic, G., Salajegheh, M., et al. (2009). Placebo-controlled trial of rituximab in lgM anti-myelin-associated glycoprotein antibody demyelinating neuropathy. Annals of Neurology, 65, 286-293.

135. Dalakas, M. C. (2018). Advances in the diagnosis, immunopathogenesis and therapies of IgM-anti-MAG antibody-mediated neuropathies. Therapeutic Advances in Neurological Disorders, 11, 1756285617746640.

136. Querol, L., \& Illa, I. (2015). Paranodal and other autoantibodies in chronic inflammatory neuropathies. Current Opinion in Neurology, 28, 474-479.

137. Querol, L., Siles, A. M., Alba-Rovira, R., et al. (2017). Antibodies against peripheral nerve antigens in chronic inflammatory demyelinating polyradiculoneuropathy. Scientific reports-UK., 7, 14411.

138. Burnor, E., Yang, L., Zhou, H., et al. (2017). Neurofascin antibodies in autoimmune, genetic, and idiopathic neuropathies. Neurology., 90, e31-e38.

139. Athanasopoulos, D., Motte, J., Fisse, A. L., et al. (2020). Longitudinal study on nerve ultrasound and corneal confocal microscopy in NF155 paranodopathy. Epub: Ann Clin Transl Neur.

140. Markvardsen, L. H., Overgaard, K., Heje, K., et al. (2018). Resistance training and aerobic training improve muscle strength and aerobic capacity in chronic inflammatory demyelinating polyneuropathy. Muscle \& Nerve, 57, 70-76.

141. Chuang, L.-L., Chen, Y.-L., Chen, C.-C., et al. (2017). Effect of EMG-triggered neuromuscular electrical stimulation with bilateral arm training on hemiplegic shoulder pain and arm function after stroke: A randomized controlled trial. Journal of Neuroengineering and Rehabilitation, 14, 122.

142. eun Jang, C., Jung, M. S., Sohn, E. H., et al. (2018). The evaluation of changes in peripheral neuropathy and quality-of-life using low-frequency electrostimulation in patients treated with chemotherapy for breast cancer: a study protocol. Trials, 19, 526 .

\section{Publisher's Note}

Springer Nature remains neutral with regard to jurisdictional claims in published maps and institutional affiliations.

Ready to submit your research? Choose BMC and benefit from:

- fast, convenient online submission

- thorough peer review by experienced researchers in your field

- rapid publication on acceptance

- support for research data, including large and complex data types

- gold Open Access which fosters wider collaboration and increased citations

- maximum visibility for your research: over $100 \mathrm{M}$ website views per year

At $\mathrm{BMC}$, research is always in progress.

Learn more biomedcentral.com/submissions 